\title{
Preferences of college girls for fabrics, colours, designs, length, flare and other designing features in one piece dresses
}

\author{
AMANPREET KAUR AND HARINDER KAUR SAGGU
}

Received: 29.08.2014; Revised: 17.11.2014; Accepted: 30.11.2014

See end of the paper for authors' affiliations

AMANPREET KAUR

Department of Apparel and

Textile Science, College of Home

Science, Punjab Agricultural

University, LUDHIANA (PUNJAB)

INDIA

Email: amudhillon1@gmail.com
ABSTRACT : For this study, a total of 90 respondents (college girls) were selected through purposive random sampling technique. Preferences of college gilrs for fabrics, colours, designs, length, flare and other designing features in one piece dresses were taken. The data collected revealed that majority of the respondents preferred georgette and satin fabric of off white colour for one piece dresses. Medium length of one piece dresses was preferred by maximum number of the respondents. It was observed that embroidery was preferred as surface embellishments by the respondents. For flare in one piece dresses pleats were given first rank by respondents.

KEY WORDS: Respondents, Preferences, One piece dresses, Surface embellishments, Flare

- HOW TO CITE THIS PAPER : Kaur, Amanpreet and Saggu, Harinder Kaur (2014). Preferences of college girls for fabrics, colours, designs, length, flare and other designing features in one piece dresses. Asian J. Home Sci., 9 (2) : 576-579. 